\title{
In Situ Analysis of Cryoformed Metals by STEM
}

Ricardo Sanson Namur ${ }^{1,2, *}$, Daniel E. Huber ${ }^{3}$, Antonio J. Ramirez ${ }^{3}$, Osvaldo M. Cintho ${ }^{1}$

1. Materials Engineering Department, State University of Ponta Grossa. Ponta Grossa, PR, Brazil.

2. Welding Engineering, Department of Materials Science and Engineering, The Ohio State University. Columbus, OH, USA.

3. Center for Electron Microscopy and Analysis, The Ohio State University, Columbus, OH, USA.

* Corresponding author: ricardonamur@hotmail.com

Processing of metals at cryogenic temperatures $(\sim 77 \mathrm{~K})$ is a well-known process that may improve mechanical properties, as strength and/or ductility. This behavior is commonly associated to the partial suppression of dynamic recovery at very low temperatures and the accumulation of crystallographic defects along the microstructure due to plastic strain [1-3]. Several studies were performed to understand the mechanisms involved in the cryogenic deformation of metals and the obtaining of ultrafine-grained and nanostructured materials [4-6]. Nevertheless, the same studies were performed by analyzing these metals at room temperature instead of the same temperature of the deformation itself, which means that important information about the structure and substructures of defects was possibly lost due to the recovery and recrystallization of these metals. Thus, in this work, the authors propose a first approach on how to analyze cryogenically deformed metals in situ, i.e., at cryogenic temperature by scanning transmission electron microscopy (STEM).

For this experiment, a small cylinder of $7 \mathrm{~mm}$ height and $6.35 \mathrm{~mm}$ thick of aluminum (Alfa Aesar 99.95\%) was used. The aluminum sample was deformed cryogenically by compression in a hydraulic press by using a polyurethane foam box filled with liquid nitrogen. After pressing and before analysis, the sample was kept under liquid nitrogen, to avoid heating and recovery. A small strip was obtained from the sample by cutting it submerged on liquid nitrogen. This small strip was then attached to a SEM stub by using a spring grade beryllium-copper clip (Fig. 1 - A). For transferring the cryogenic deformed sample to the microscope, a PP3010T Cryo-SEM preparation system was used (Fig. 1 - B). The sample stage of the microscope was cooled down during the whole process.

A foil was successfully obtained by FIB processing of the material using a Helios 600 DualBeam FIBSEM with a Quorum PPT 3010 cryo preparation system. In situ lift out of the foil, was performed using an Omniprobe AutoProbe 200 system. STEM Imaging was conducted with the specimen on the needle and while cooling the needle. The method of cooling the needle was developed by the authors. STEM imaging of the sample was obtained by using a solid state STEM diode fixed to the microscope stage. Figure 2 presents an image obtained by STEM for the aluminum sample. The evidence of electron transmission at $30 \mathrm{Kv}$ indicates that the foil was successfully thinned by this method, allowing the observation of contrast between adjacent grains. The image obtained by STEM shows that the microstructure obtained after cryogenic deformation is highly refined, as reported in literature.

The results from this preliminary research confirm that in-situ cryo FIB-SEM STEM is a promising technique for analyzing, the microstructure of metals deformed cryogenically, thus, understanding the mechanisms involved in the microstructural evolution of these materials after heating. 


\section{References:}

[1] DCC Magalhães, et al, Journal of Materials Science 52 (2017), p. 7466.

[2] MY Maeda, et al, Materials Research 20 (2017), p. 716.

[3] YL Gong, et al, Materials Science and Engineering A 583 (2013) p. 199.

[4] MA Meyers, O. Vohringer, V.A. Lubarda, Acta Materialia 49 (2001) p. 4025.

[5] T Konkova, et al, Acta Materialia 58 (2010) p. 5262.

[6] T Konkova, et al, Scripta Materialia 63 (2010) p. 921.
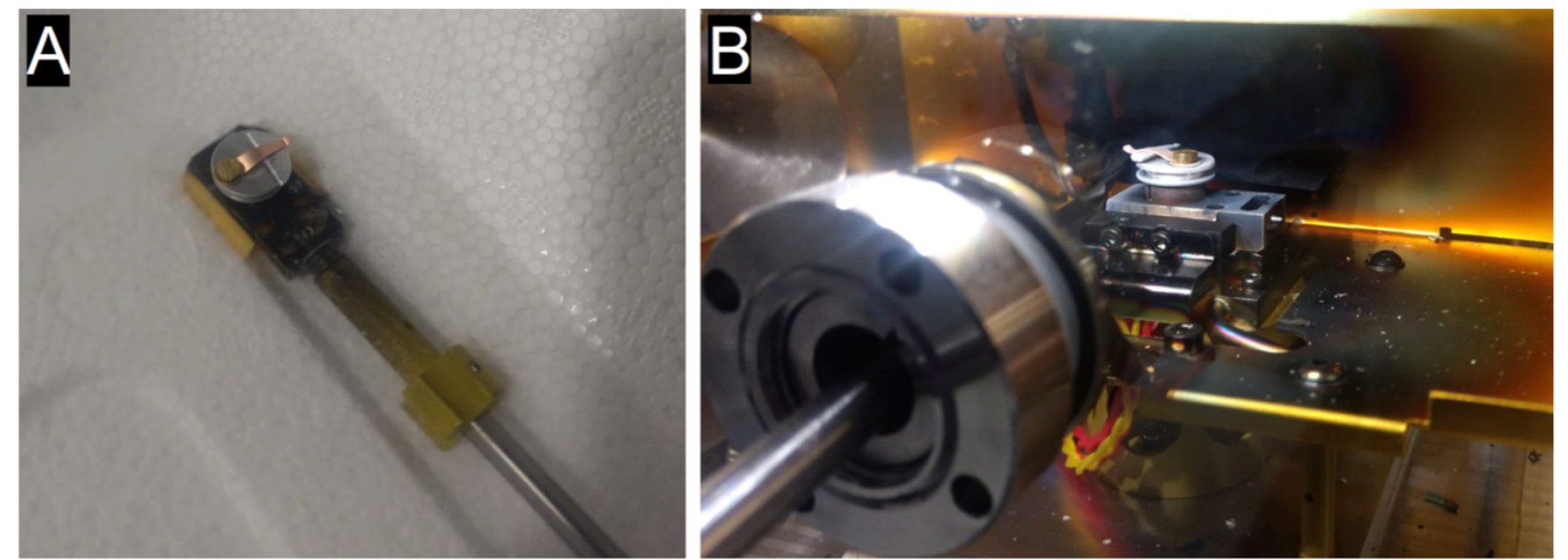

Figure 1. In (A), the aluminum sample attached to the samples holder by a beryllium-copper clip. In (B), the sample being transferred to the microscope chamber by using a Cryo-SEM transfer system.

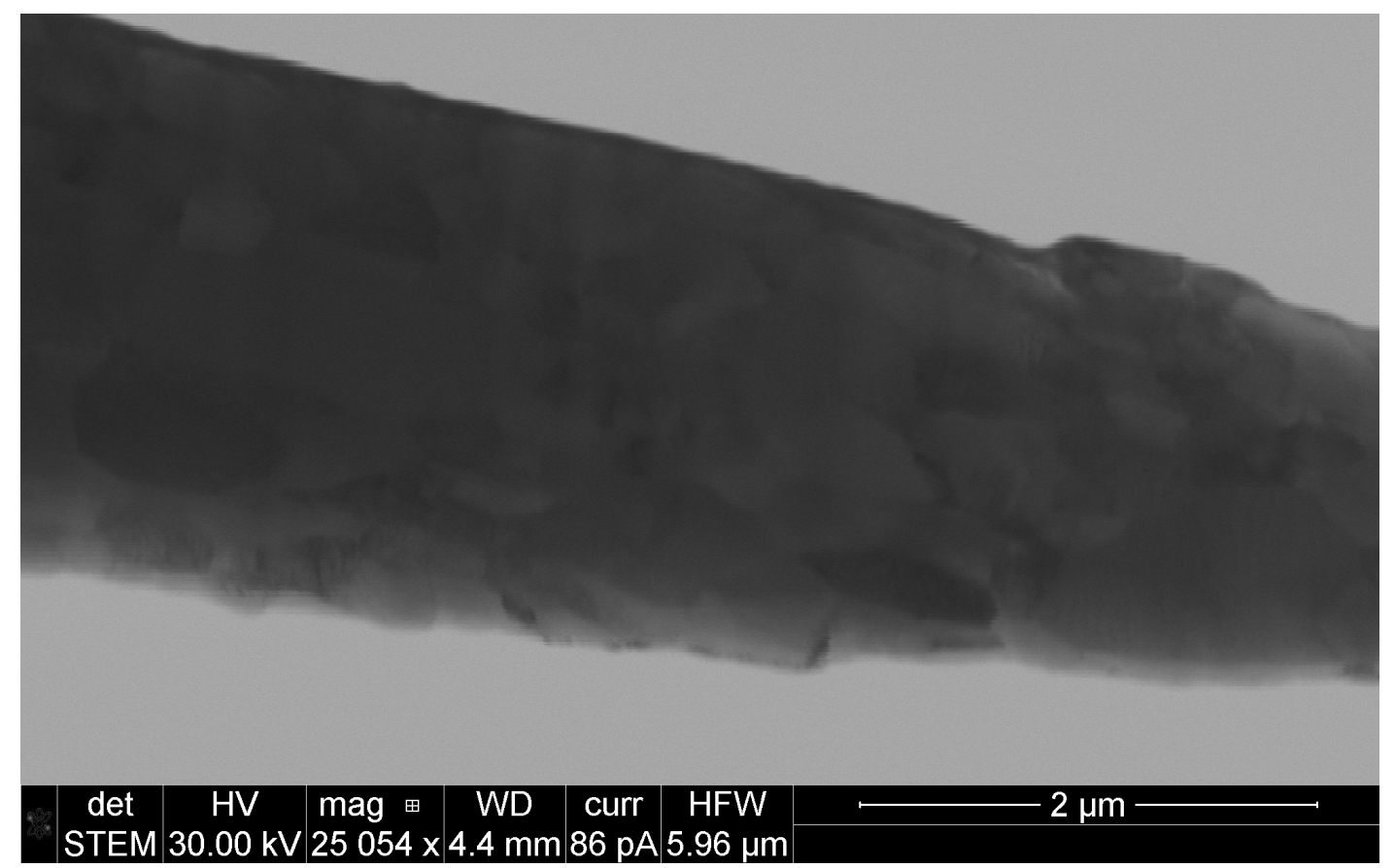

Figure 2. STEM image for the aluminum foil obtained by FIB after cryogenic deformation. Contrast between grains indicate a highly refined microstructure. 